\section{False Engagements in AFM}

Chetan Dandavate, Ohio State University

In scanning microscopes, like the Atomic Force Microscope (AFM), used in contact mode, scanning begins with engaging the tip with the sample at some contact force, which can be adjusted by the setpoint" (this is common to Digital Instruments' AFMs). It may differ for other brands. For a system that detects the motion of the cantilever with a laser beam, the setpoint basically gives an idea of the voltage difference between the top and bottom photo detectors. When the tip comes into contact, the feedback circuit adjusts the tip deflection according to the required contact force. This is the method commonly followed for the constant deflection method.

False engagement happens before the tip comes into contact with the sample, if for some reason the difference between top and bottom photo detectors during tip lowering reaches the setpoint. Because of this, the feedback circuit "thinks" that the tip is already in contact and begins the scan. This can be seen either by viewing the topography image, which is perfectly plain, or by going to the force curve mode, where the standard curve cannot be obtained unless the tip is advanced.

In the Nanoscope III family of AFM's, it can be seen that the difference signal of the top/bottom detector goes on increasing as the tip approaches the sample. One reason could be that the sample is reflective, and so light is being reflected onto the detector in such a way that as the tip is bought closer, the setpoint is reached, even if the tip is not in contact. However, false engagement has been seen to be more common when a cantilever of lower stiffness is used (for example using silicon nitride cantilevers of force constant $0.06 \mathrm{~N} / \mathrm{m}$ while imaging silicon). This gives some reason to believe that the cantilever is being deflected because of interatomic forces. The best remedy for this would be to use a cantilever of higher stiffness $(0.6 \mathrm{~N} / \mathrm{m})$ or if

\section{Take the following microanalysis quiz}

\author{
What is the thickness of my film? \\ Does the beam penetrate that particle? \\ What is the best $k V$ to use for this sample? \\ How wide is the beam in my E-SEM? \\ How much does an incorrect analysis cost? \\ How can I improve the quality of my analysis?
}

\section{Maybe it's time to take a look at the software that can answer these questions}

\section{Electron Flight Simulator}

Analysis Simulation and Modeling Software for Windows

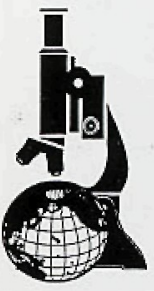

\section{Small World}

phone/fax: (703) 849-1492 e-mail: dchernoff@aol.com http://www.small-world.net that's not possible, to keep the setpoint sufficiently high enough to prevent the false engagement. This could be detrimental if the sample is soft.

To minimize damage, decrease the scan size to minimum, engage the tip and then offset to the required scan size, scanning an area that does not include the point of contact.

"Note:

In an AFM, the motion of the cantilever is detected by the change in the photo diode detector signal. Typically, it consists of a four quadrant area which is highly sensitive to the position of the light falling on it and the signal which we use to detect vertical motion of the cantilever is the difference in signal between the top two quadrants and the bottom two quadrants. This is denoted by a num- त ber, the setpoint, which generally ranges from $-10 \mathrm{~V}$ to $+10 \mathrm{~V}$. During engaging, when we specify the setpoint, we give a value which is slightly higher than the current value (free air) the detector shows (because the Voltage signal increases or becomes more positive as the cantilever deflects due to normal contact force acting outwards from the sample). When the cantilever engages, the feedback mechanism will adjust the cantilever deflection so that it conforms to the specified setpoint, which if higher than the free air voltage, will mean that the cantilever is being deflected because of normal contact forces. Hence, in this case, there will be normal contact force interactions between the tip and sample.

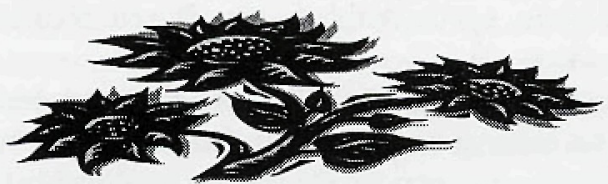

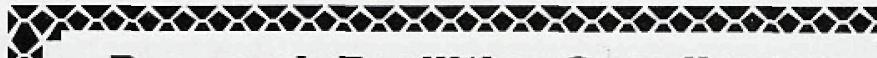 Research Facilities Coordinator}

The Department of Anatomy and Neurobiology at the University of Vermont College of Medicine seeks a Research Facilities Coordinator to supervise the daily operation of a shared Noran-based laser scanning confocal imaging system.

The individual will provide technical support and/or collaborate on projects from a number of different laboratories in addition to providing training to faculty, postdoctoral fellows and graduate students. There also will be an opportunity to develop independent research projects. The individual will be responsible for equipment maintenance, upgrading hardware and software as required scheduling and billing.

Preference will be given to candidates with documented theoretical and practical experience with imaging techniques, use of fluorescent dyes and diverse experience with UNIX, DOS and Mac computer operating systems. Strong communication and interpersonal skills are needed along with the ability to manage multiple projects simultaneously.

Minimal requirements are a BS degree and a number of years of experience with confocal and other forms of microscopy. MS or Ph.D. degrees in a biological or physical science will be beneficial. Position classification and salary are negotiable and commensurate with training and experience.

Send CV and the names of three references to:

Dr. Rodney L. Parsons,

Department of Anatomy and Neurobiology,

University of Vermont College of Medicine, C427 Given Building,

Burlington, Vermont 05405.

Email: rparsons@zoo.uvm.edu.

Applications from women and minorities are encouraged.

The University of Vermont Is an equal opportunity employer. 
- Quartz Halogen Light Sources

- Ringlights - Standard and ESD Safe

- Dual and Single Self-Supporting

Light Guides

- Microscope Accessories

- Fast Delivery

- Aggressive Pricing

- Innovative Products and Accessories

- Custom and OEM Capabilities

- Quality Products for over 35 years - Lifetime Warranty

\section{See our website for NEW product information!}

Attention Microscope Dealers:

Dolan-Jenners is interested in growing our distribution through building relationships with microscope dealers.

Please call Gus Vassiliades at ext. $\mathbf{3 0 1 6}$ for more information.

\section{Fiber Optic Illumination} for Microscopy

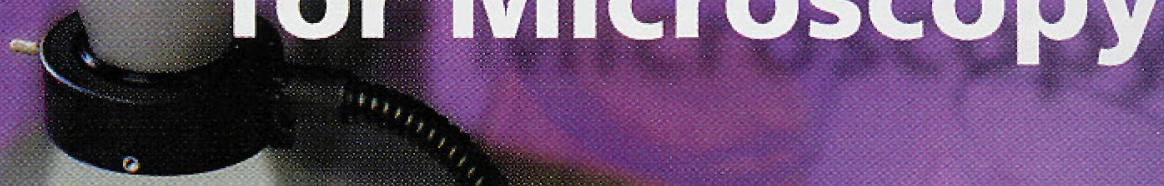

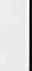

